\title{
Mu Suppression as an Indicator of Activation of the Perceptual- Motor System by Smoking-Related Cues in Smokers
}

\author{
Cheryl L. Dickter \\ William \& Mary, cldickter@wm.edu \\ Paul D. Kieffaber \\ Julie A. Kittel \\ Catherine A. Forestell \\ William \& Mary, caforestell@wm.edu
}

Follow this and additional works at: https://scholarworks.wm.edu/aspubs

Part of the Psychology Commons

\section{Recommended Citation}

Dickter, Cheryl L.; Kieffaber, Paul D.; Kittel, Julie A.; and Forestell, Catherine A., Mu Suppression as an Indicator of Activation of the Perceptual-Motor System by Smoking-Related Cues in Smokers (2013). Psychophysiology, 50(7), 664-670. https://doi.org/10.1111/psyp.12044

This Article is brought to you for free and open access by the Arts and Sciences at W\&M ScholarWorks. It has been accepted for inclusion in Arts \& Sciences Articles by an authorized administrator of W\&M ScholarWorks. For more information, please contact scholarworks@wm.edu. 
This is the peer reviewed version of the following article: Dickter, C. L., Kieffaber, P. D., Kittel, J. A., " Forestell, C. A., (2013). Mu Suppression as an Indicator of Activation of the Perceptual-Motor System by Smoking-related Cues in Smokers. Psychophysiology, 50 (7), 664-70. doi: 10.1111/psyp.12044, which has been published in final form at doi: 10.1111/psyp.12044. This article may be used for non-commercial purposes in accordance with Wiley Terms and Conditions for Use of Self-Archived Versions

\rrh\Mu suppression in smokers

Urh\C.L. Dickter et al.

lalMu suppression as an indicator of activation of the perceptual-motor system by smokingrelated cues in smokers

IbICHERYL L. DICKTER, PAUL D. KIEFFABER, JULIE A. KITTEL, AND CATHERINE A. FORESTELL

IclDepartment of Psychology, Integrated Science Center, The College of William \& Mary, Williamsburg, Virginia, USA

IfnlThe authors would like to thank Jason Wright for his assistance with data collection and Madison Crutcher for her help with data analysis.

Address correspondence to: Cheryl L. Dickter, Ph.D., Department of Psychology, Integrated Science Center, College of William \& Mary, Williamsburg, VA 23187. E-mail: cldickter@wm.edu 


\section{le\Abstract}

The goal of the current study was to determine whether activation of the mirror neuron system, as measured by mu rhythm desynchronization, varied as a function of image content in smokers compared with nonsmokers. EEG activity was recorded while participants passively viewed images depicting smoking-related and nonsmoking-related stimuli. In half of the images, cues were depicted alone (inactive), while for the remaining images, cues were depicted with humans interacting with them (active). For the nonsmoking stimuli, smokers and nonsmokers showed greater mu suppression to the active cues compared to the inactive cues. However, for the smoking-related stimuli, active cues elicited greater perception-action coupling in smokers compared to nonsmokers as reflected in their enhanced mu suppression. The results of the current study support the involvement of the perceptual-motor system in the activation of motivated drug use behaviors.

Descriptors: $\mathrm{Mu}$ rhythm desynchronization, Mirror neurons, Smoking, Addiction, Tobacco 
Cigarette smoking is the leading preventable cause of premature death in the United States (National Institutes of Health, 2012). Given the impact that nicotine has on health outcomes, Q3 it is important to understand the nature of drug addiction. From a biological perspective, it is now generally accepted that repeated exposure to drugs such as nicotine results in persistent and complex changes in neural circuitry that controls responses to rewards and regulates motivated behavior (Berridge \& Robinson, 1993). One mediator of drug reward is the mesolimbic dopamine system (Koob, Caine, Markou, Pulverinti, \& Weiss, 1994). These dopaminergic neurons have cell bodies in the ventral tegmental area of the midbrain and project to areas within the limbic forebrain including the nucleus accumbens. Sensitization of this neural circuitry is thought to result in the attribution of incentive salience both to drugs and to the stimuli with which those drugs are associated (see Berridge, 2007, for a review).

Sensitization to drug-related stimuli can be manifested as an increase in the allocation of attentional resources to the drug and its associated paraphernalia, which in turn increases the frequency of drug-related cognitions and diminishes the attentional resources available for other environmental cues. Such attentional biases to drug-related cues in addicts have been demonstrated using implicit reaction time paradigms (e.g., Field \& Cox, 2008; Mogg, Bradley, Field, \& de Houwer, 2003; Townshend \& Duka, 2001; Vadhan et al., 2007). In the case of nicotine addiction, an increase in attentional bias to smoking cues has been reported for daily smokers (Waters \& Feyerabend, 2000; Waters, Shiffman, Bradley, \& Mogg, 2003). These Q4 findings have also been supported by electophysiological results through the measurement of electroencephalograph (EEG). As demonstrated in a recent meta-analysis by Littel, Euser, Munafò, and Franken (2012), the P3 response, which is thought to reflect attentional Q5 allocation to motivationally salient stimuli (Polich, 2012; Polich \& Kok, 1995), is enhanced to 
drug-related stimuli in addicts compared to controls (e.g., Ceballos, Bauer, \& Houston, 2009). With respect to nicotine, most studies have demonstrated that smokers consistently have a larger P3 amplitude to smoking-related stimuli relative to nonsmokers (Littel \& Franken, 2007, 2011; but see Warren \& McDonough, 1999).

Although behavioral and electrophysiological studies suggest that attention plays an important role in the initiation and maintenance of addiction, comparatively less is known about how smoking-related cues may be processed by the perceptual-motor system. Activation of the mirror neuron system has been shown to be associated with the observation and/or performance of goal-directed actions and is commonly measured over the sensorimotor cortex in humans using EEG (see Pineda, 2005, for a review). Specifically, activation of the mirror neuron system is thought to be indexed by suppression of oscillatory activity in the frequency range $8-13 \mathrm{~Hz}$ and is called the mu rhythm. Participants viewing individuals reaching towards objects show a suppression of the mu rhythm (Muthukumaraswamy, Johnson, \& McNair, 2004), as do participants viewing a video of others tossing an object towards a camera (Oberman, Pineda, \& Ramachandran, 2007). Suppression or "desynchronization" of the mu rhythm has also been shown to occur in cases in which "implied" motion is observed wherein goal-directed actions are inferred from static images (Urgesi, Moro, Candidi, \& Aglioti, 2006).

Studies of mu suppression have also been used to advance our understanding of human imitation and feelings of empathy towards others. For example, females have been shown to demonstrate more mu suppression than males while observing painful situations (Yang, Decety, Lee, Chen, \& Cheng, 2009), and autistic individuals show less mu suppression than matched controls (Oberman et al., 2005). Higher levels of mu suppression in these studies are thought to reflect higher levels of empathy and social competency. 
The goal of the current study was to determine whether smoking-related stimuli presented in a social context (e.g., other people holding a cigarette or actively smoking) differentially activates perception-action coupling in the mirror neuron system in smokers and nonsmokers. Such a finding could have important implications because activation of perceptual-motor coupling in individuals who smoke may motivate behaviors related to drug addiction and may help to explain how drug use is perpetuated. To date, only one published study (Pineda \& Oberman, 2006) has examined mu suppression to smoking-related stimuli. In this study, participants viewed videos of a hand pulling a cigarette out of a package (i.e., smoking cue) and a hand pulling a crayon out of a package (i.e., nonsmoking cue) and observed their own hands in similar motions. Results indicated that, although nonsmokers showed the expected mu suppression to observed and self-generated hand movements in the videos, smokers did not exhibit mu suppression when observing others' hand movements in the videos, regardless of whether they were interacting with smoking or nonsmoking cues. The authors suggested that this finding supports the idea that addiction changes the way the mirror neuron system is activated by drug-related cues. However, more research is needed to understand the mirror neuron system's role in processing addiction-related cues.

In the current study, EEG activity was recorded while participants passively viewed a series of images depicting either a smoking-related stimulus (e.g., a cigarette) or a nonsmokingrelated stimulus (e.g., a toothbrush). Half of the smoking-related images and the nonsmokingrelated images depicted pictures of the cue alone (i.e., inactive cues), while the remaining images depicted a human interacting with the cue (i.e., active cues). We hypothesized that smokers and nonsmokers would show greater mu suppression to the active compared to inactive nonsmoking stimuli, as the mu rhythm has been shown to be sensitive to implied human movements in static 
images (Urgesi et al., 2006). For the smoking stimuli, however, it was hypothesized that active smoking-related stimuli would elicit greater suppression of the mu rhythm in smokers relative to nonsmokers. Given the absence of implied action, it was expected that smokers and nonsmokers would show similarly low levels of mu suppression to the inactive smoking cues.

\section{\1\Method}

\section{2\Participants}

Fifty-one undergraduates between the ages of 17 and 22 years at a medium-sized liberal arts college were recruited through an online database and provided with credit in their introductory psychology course or recruited through advertisements and paid $\$ 10$ for their participation. Participants were asked to refrain from smoking a cigarette for at least $2 \mathrm{~h}$ before their scheduled session if they were smokers and were told that their carbon monoxide levels would be measured to ensure compliance. All participants were healthy young adults with normal or corrected-tonormal vision and were right-handed with no history of head trauma. All procedures were approved by the Protection of Human Subjects Committee, and written informed consent was obtained from each participant.

\section{LMaterials}

13\Stimuli. The experimental stimuli consisted of 40 color photographs; as depicted in Figure 1|f1\, half of these were smoking related and half were nonsmoking related (see Dickter \& Forestell, 2012; Forestell, Dickter, Wright, \& Young, 2011, for examples of color stimuli). Half of the pictures were active in that they depicted a person interacting with the stimulus, whereas the remaining pictures were inactive, in that they consisted of the stimulus alone. The nonsmoking-related photographs were created to be similar to the smoking-related photographs on various dimensions including color, brightness, and object orientation. To control for 
potential laterality effects, half of the pictures in each of the active conditions depicted the right side of the body (e.g., right hand holding a cigarette) and the other half depicted the left side of the body. All images were successfully pilot-tested with 10 nonsmoking undergraduates to ensure that they could identify the image content and correctly judge whether or not they were drug related. The average accuracy rate for the stimuli was $98 \% \pm 0.08$ (range: $90 \%-100 \%$ ). Picture stimuli are available upon request from the corresponding author.

\3\Questionnaires. In addition to demographic questions about participants' age, ethnic and racial background, family income, and parents' level of education, participants self-reported their smoking history in an online questionnaire. Questions included whether the participant had ever smoked and, if so, the age of their first cigarette and the number of cigarettes they typically smoked per day and per week. Smokers also filled out the following validated questionnaires. The Michigan Nicotine Reinforcement Questionnaire (MNRQ; Pomerleau, Fagerström, Marks, Tate, \& Pomerleau, 2003) is a 13-item scale that measures the amount of positive Q6 and negative reinforcement that smokers gain from their nicotine addiction. Positive reinforcement (five questions; $\alpha=.79$ ) focuses on the pleasurable effects obtained from smoking, and includes items such as "I smoke because it is pleasurable." Negative reinforcement (eight questions; $\alpha=0.84$ ) focuses on nicotine withdrawal symptoms, and includes items such as "At times when I have been unable to smoke due to restrictions on smoking or because I was trying to quit, I experienced trouble falling asleep to the following extent." Response choices for each question in the MNRQ were on a 4-point scale.

The Questionnaire of Smoking Urges-Brief (QSU-B; Tiffany \& Drobes, 1991; $\alpha=.97$ ) was included to measure nicotine craving in smokers. It has 10 questions and dichotomizes craving into two factors: reward and relief. Reward craving consists of an intention to smoke, 
with the anticipation that some pleasure will be derived from smoking. Relief craving consists of an intense desire to smoke, with the anticipation that smoking will relieve symptoms related to withdrawal.

13\Carbon monoxide monitor. A carbon monoxide (CO) BreathCO monitor (Vitalograph, Lenexa, KS) was used to assess recent tobacco smoke exposure.

\section{2\Procedure}

Upon arriving at the laboratory, participants completed a consent form, provided a CO reading, and were seated in an electrically shielded Faraday chamber approximately $70 \mathrm{~cm}$ from a computer monitor. Participants were asked to be as still as possible during the experiment in order to reduce the amount of extraneous noise in the EEG recordings. All participants were told that the computer task involved the presentation of a series of trials, each composed of a picture. They were instructed to pay attention to the pictures because they would be asked about them later. Participants completed the experimental trials, in which the 40 pictures were each presented in a random order for each of three blocks of trials, with short breaks in between each block. Therefore, participants viewed 120 trials in total. Each picture was displayed on the screen for $8 \mathrm{~s}$, followed by an intertrial interval of $8 \mathrm{~s}$. After completion of the EEG task, participants completed the questionnaires online. When finished, they were debriefed and given credit for their participation. All participants completed the study within $1.5 \mathrm{~h}$.

\section{2\Electrophysiological Recording and Analysis}

EEG data were recorded at a rate of $1000 \mathrm{~Hz}$ using a DBPA-1 high-impedance bioamplifier (Sensorium Inc., Charlotte, VT) with an analog high-pass filter of $0.01 \mathrm{~Hz}$ and a low-pass filter of $500 \mathrm{~Hz}$ (four-pole Bessel). The EEG was recorded from $74 \mathrm{Ag}-\mathrm{AgCl}$ sintered electrodes in an electrode cap, placed using the expanded International 10-20 electrode placement system. All 
electrodes were referenced to the tip of the nose, and the ground electrode was placed in the middle of the forehead, slightly above the eyebrows. Eye movement and blinking were recorded from electrodes placed on the lateral canthi and perioccular electrodes on the superior and inferior orbits, aligned with the pupils. Before data collection was initiated, all impedances were adjusted to within 0-20 kilohms. EEG was recorded continuously throughout the task, and was analyzed offline using EEGLAB. Data were undersampled at $500 \mathrm{~Hz}$. The data were corrected for eye movement artifacts, using independent component analysis (Jung et al., 2000). Channels containing extreme values $( \pm 300 \mathrm{mV})$ in more than $40 \%$ of the sweeps were automatically marked for visual inspection and spatially interpolated where appropriate. All EEG data were low-pass filtered (FIR) with a half-amplitude of $20 \mathrm{~Hz}$ (Luck, 2005).

\section{$\backslash 1 \backslash$ Results}

\section{2\Participant Characteristics}

Of the 51 participants who were recruited, seven were excluded because of missing data, one was excluded because of excessive artifacts in their EEG data, and one was excluded because he had quit smoking. All smokers confirmed that they had refrained from smoking for the previous $2 \mathrm{~h}$. Based on their reported smoking behavior, participants were divided into two groups: smokers $(n=22)$ who reported that they smoked between 4-5 cigarettes per day, and nonsmokers who reported that they had either never smoked a cigarette $(n=18)$, or smoked fewer than 4 cigarettes in their lifetime but none in the past year $(n=2)$. As shown in Table $1 \backslash t 1 \backslash$, smokers who had been smoking for about 3 years had an average CO reading between 3 and 4 ppm, which was significantly higher than that of nonsmokers; $F(1,40)=6.85, p<.02$. Moreover, smokers were collectively about one year older than the nonsmokers, $F(1,40)=6.85, p<.02$. 
A time-frequency analysis was performed using EEGLAB (Delorme \& Makeig, 2004) to characterize mu suppression over the 8-s interval following target presentation. Event-related spectral perturbation (ERSP) was computed in EEGLAB using Morlet wavelets over segments of data spanning $-7,000$ to $8,000 \mathrm{~ms}$ relative to stimulus onset from electrodes $\mathrm{C} 3$ and $\mathrm{C} 4$ for each trial. These electrodes were selected because they have been demonstrated to be the most robust locations for measurement of the mu rhythm in topographical studies (McFarland, Minder, Q7 Vaughan, \& Wolpaw, 2000) and because they are the most commonly used locations for measurement of the mu rhythm in studies of children (Marshall \& Meltzoff, 2011), the development of brain-computer interfaces (Pineda, Silverman, Vankov, \& Hestenes, 2003), and in studies of social cognition (Pineda \& Hecht, 2008). A 5-s interval between -6,000 ms and $1,000 \mathrm{~ms}$ with respect to target image onset was used for baseline normalization. The wavelet analysis was performed over frequencies between 2 and $75 \mathrm{~Hz}$. The "cycles" parameter in EEGLAB was set to [3 .5] such that the number of significant wavelet cycles was $\sim 3$ at 2 Q11 $\mathrm{Hz}, \sim 56$ at $75 \mathrm{~Hz}$, and $\sim 12$ over the mu frequency band. The wavelets spanned approximately $1,500 \mathrm{~ms}$ over the mu frequency band and were overlapped by $95 \%$, yielding a temporal resolution of $69 \mathrm{~ms}$ in the time-frequency plane.

Grand-averaged ERSPs were computed separately for active and inactive target stimuli, revealing a selective decrease in ERSP band power following active, but not inactive stimuli, as illustrated in Figure 2\f2\. Based on this analysis of the active-inactive differences, mu suppression was measured between 700 and 6,000 ms following target presentation. Thus, mu desynchronization was quantified as the average ERSP (dB-scaled) over the mu $(8-13 \mathrm{~Hz})$ frequency range, averaged over the 700-6,000 ms poststimulus epoch and electrodes C3 and C4. 
To examine the effects of image content and type of stimulus on the mirror neuron systems in smokers and nonsmokers, this measure of mu suppression was submitted to a 2 (Stimulus Type: smoking vs. nonsmoking) $\times 2$ (Image Content: active vs. inactive $) \times 2$ (Smoking Status: smoker vs. nonsmoker) mixed model analysis of variance (ANOVA) with repeated measures on the first two factors. This analysis revealed a main effect of image content, such that suppression was greater for active stimuli $(M=-1.20, S E=0.12)$ compared to inactive stimuli $(M=-0.80, S E=0.10), F(1,40)=20.91, p<.001, \eta^{2}=.34$. In addition, there was an Image Content $\times$ Smoking Status interaction, $F(1,40)=4.89, p<.04, \eta^{2}=.11$, demonstrating that smokers showed greater suppression for active stimuli $(M=-1.46, S E=0.16)$ compared to inactive stimuli $(M=-0.85, S E=0.12), F(1,21)=24.86, p<.001, \eta^{2}=.54$, whereas nonsmokers showed similar mu suppression for active $(M=-1.46, S E=0.16)$ and inactive cues $(M=-0.85$, $S E=0.12), F(1,19)=2.58, p=.125, \eta^{2}=.12$. There was also a significant Image Content $\times$ Stimulus Type interaction, $F(1,40)=6.88, p<.02, \eta^{2}=.15$, which revealed that mu suppression was greater for active smoking cues $(M=-1.19, S E=0.14)$ compared to inactive smoking cues $(M=-0.94, S E=0.12), F(1,41)=4.33, p<.05, \eta^{2}=.10$, and mu suppression was greater for active nonsmoking stimuli $(M=-1.25, S E=0.12)$ than inactive nonsmoking stimuli $(M=-0.66$, $S E=0.11), F(1,41)=25.94, p<.001, \eta^{2}=.39$.

All of these effects were qualified by a marginal Stimulus Type $\times$ Image Content $\times$ Smoking Status interaction, $F(1,40)=3.14, p<.08, \eta^{2}=.07 .{ }^{1} \backslash$ fn $1 \backslash$ Sub-ANOVAs examining image content and smoking status were conducted to break down the three-way mu suppression interaction by stimulus type (i.e., smoking pictures were analyzed separately from nonsmoking pictures). As shown in Figure $3 \backslash \mathrm{f} 3 \backslash$, for the smoking images there was a significant Image Content $\times$ Smoking Status interaction, $F(1,41)=4.33, p<.05, \eta^{2}=.10$, such that smokers 
demonstrated more suppression to the active smoking-related pictures $(M=-1.52, S E=.18)$ than for the inactive smoking-related pictures $(M=-1.39, S E=.17), F(1,21)=20.74, p<.001, \eta^{2}=$ .50 , whereas nonsmokers did not $(p>.60)$. When similar analyses were conducted for the nonsmoking images, only a main effect of image content was revealed, such that greater suppression occurred in response to the active images $(M=-1.24, S E=.12)$ compared to the inactive images $(M=-0.65, S E=.11), F(1,37)=25.20, p<.001, \eta^{2}=.387$.

Correlations were additionally conducted to further understand which factors may be related to mu suppression in smokers. These analyses included the subscales from the QSU-B and MNRQ, as well as various other characteristics of smokers such as the number of years they smoked, their CO reading, and their mu suppression means for each stimulus category. These analyses revealed a significant positive correlation between QSU-B relief and mu suppression for the active smoking stimuli, $r(21)=0.55, p<.01$, indicating that as QSU-B relief scores increased, mu suppression decreased. Additionally, there was a marginally significant positive correlation between QSU-B reward and mu suppression to active smoking stimuli, $r(21)=0.39$, $p<.08$. These subscales did not correlate significantly with mu suppression for any of the other stimulus categories. Moreover, the remaining measures did not significantly correlate with mu suppression for any of the stimulus categories.

\section{Viscussion}

The current study is the first to demonstrate differential mu rhythm desynchronization between smokers and nonsmokers in response to images that depict implied action toward smokingrelated cues. As hypothesized, smokers demonstrated greater perception-action coupling than nonsmokers in response to the smoking-related stimuli containing human content (active cues) relative to inactive cues as reflected in enhanced mu suppression. In contrast, smokers and 
nonsmokers showed greater mu suppression to the nonsmoking active cues compared to the nonsmoking inactive cues, reflecting similar activation of the mirror neuron system to implied movement toward nonsmoking cues in both groups.

Previous behavioral work has demonstrated that smokers and nonsmokers process smoking-related cues with and without human content differently (Dickter \& Forestell, 2012; Forestell et al., 2011). Specifically, smokers show a greater attentional bias to inactive smokingrelated cues compared to nonsmokers, but there is no difference between the groups in response to active smoking-related cues. The current work extends these behavioral findings by demonstrating that smokers experience dissociable neural responses in the mirror neuron system when exposed to images that depict humans interacting with smoking-related cues. It is important to note, however, that as this study is one of the first to measure the role of the mirror neuron system in addiction, these results should be interpreted with caution. Replicating these effects as well as examining whether smokers' responses to these active cues are a function of empathetic responses to other smokers (see Oberman et al., 2005; Yang et al., 2009) should be goals of further research. An alternative interpretation of the findings reported herein is that nonsmokers demonstrate diminished mu desynchronization to active smoking cues. This contention is based on the observation that nonsmokers' mu suppression was similar to the active and inactive smoking cues, despite the fact that the active pictures contained implied goaldirected actions. Such an interpretation would suggest that these smoking-related stimuli are not motivationally relevant to nonsmokers.

It should be noted that the current results differ from those reported by Pineda and Oberman (2006). They reported that, although smokers exhibited normal suppression to selfmovement, they did not demonstrate suppression to movements of others interacting with either 
addiction-related cues or control cues, suggesting that overall mu suppression to others' goaldirected movements may be impaired in smokers. The inconsistencies between our findings and those of Pineda and Oberman may have occurred because of differences between our samples. While Pineda and Oberman's sample consisted of smokers who reported smoking 13-14 cigarettes per day, our sample consisted of participants who smoked 4-5 cigarettes per day. Together, the results of these studies suggest that while light smokers demonstrate mu suppression to pictures of others interacting with smoking-related cues, heavy smokers' overall mu suppression to others' actions may be reduced and not differentially activated by the presence or absence of smoking-related cues. This contention is further supported by our finding that those with higher QSU-B relief (and to a lesser extent QSU-B reward) scores demonstrated reduced mu suppression for the active smoking-related cues. Future research should include a measure of dependence to determine whether it correlates with mu suppression to active smoking cues in smokers.

The results of the current study provide evidence for the involvement of the perceptualmotor system in the activation of motivated behaviors related to drug use and for the importance of considering the context in which drug-related cues are perceived. These results provide insight into the specific social cognitive processes associated with drug use and, consistent with contemporary models of addiction (i.e., Berridge \& Robinson, 1993; Franken, 2003), suggest that drug use and addiction may be perpetuated by fundamental changes in the neural processing of drug-related stimuli (Tiffany, 1990). Whether levels of mu suppression to active smokingrelated stimuli can be used to predict the likelihood of long-term smoking or the success of quit attempts is a fruitful area for future research. A number of questions also remain concerning the functional significance of mirror neuron activity to substance-related cues. In particular, it is 
unknown whether substance users who are at elevated risk for the development of abuse or dependence, but who have yet to develop a substance use disorder, demonstrate heightened mu suppression to active substance-related cues. This issue is important for understanding whether the enhanced mirror neuron activity elicited by active drug cues is a precursor to or a consequence of drug abuse. 


\section{Footnote}

$\backslash f n \backslash 1$. To determine the specificity of this effect, identical analyses were performed for frontal (F3/F4) and parietal (P3/P4) sites, and additional analyses for gamma $(25-75 \mathrm{~Hz})$ and theta $(4-7 \mathrm{~Hz})$ frequency bands were performed for frontal, central, and parietal sites. In all cases, the three-way interaction between stimulus type, image content, and participant status was nonsignificant. 


\section{\1\References}

Iref\Anokhin, A. P., Vedeniapin, A. B., Sirevaag, E. J., Bauer, L. O., O’Connor, S. J., Kuperman, $\begin{array}{ll}\text { S., ... . (2000). The P300 brain potential is reduced in smokers. } & \text { Q2, Q9 }\end{array}$ Psychopharmacology, 149, 409-413. doi: 10.2007/s002130000387

Berridge, K. C. (2007). The debate over dopamine's role in reward: The case for incentive salience. Psychopharmacology, 191, 391-431. doi: 10.1007/s00213-006-0578-x

Berridge, K. C., \& Robinson, T. E. (1993). The neural basis of drug craving: An incentivesensitization theory of addiction. Brain Research Reviews, 18, 247-291. doi: 10.1016/0165-0173(93)90013-P

Ceballos, N. A., Bauer, L. O., \& Houston, R. J. (2009). Recent EEG and ERP findings substance abusers. Clinical EEG Neuroscience, 20, 122-128. doi: 10.1177/155005940904000210

Delorme, A., \& Makeig, S. (2004). EEGLAB: An open source toolbox for analysis of single-trial EEG dynamics including independent component analysis. Journal of Neuroscience Methods, 134, 9-21. doi: 10.1016/j.jneumeth.2003.10.009

Dickter, C. L., \& Forestell, C. A. (2012). Peering through the smoke: The effect of parental smoking behavior and addiction on daily smokers' attentional bias to smoking cues. Addictive Behaviors, 27, 187-192. doi: 10.1016/j.addbeh.2011.09.017.

Fabiani, M., Gratton, G., \& Federmeier, K. D. (2007). Event-related brain potentials: Q9 Methods, theory, and applications. In J. T. Cacioppo, L. Tassinary, \& G. G. Berntson (Eds.), Handbook of psychophysiology (3rd ed., pp. 85-119). Cambridge, UK: Cambridge University Press. 
Field, M., \& Cox, W. M. (2008). Attentional bias in addictive behaviors: A review of its development, causes, and consequences. Drug and Alcohol Dependence, 97, 1-20. doi: 10.1016/drugalcdep.200803.030

Forestell, C. A., Dickter, C. L., Wright, J. D., \& Young, C. M. (2011). Clearing the smoke: Parental influences on non-smokers' attentional biases to smoking-related cues. Psychology of Addictive Behaviors, 26, 638-643. doi: 10.1037/a0025096.

Franken, I. H. A. (2003). Drug craving and addiction: Integrating psychological and neuropsychopharmacological approaches. Progress in Neuro-Psychopharmacology and Biological Psychiatry, 27, 563-579. doi: 10.1016/S0278-5846(03)00081-2.

Jung, T. P., Makeig, S., Westerfield, M., Townsend, J., Courchesne, E., \& Sejnowski, T. J. (2000). Removal of eye activity artifacts from visual event-related potentials in normal and clinical subjects. Clinical Neurophysiology, 111, 1745-1758. doi: 10.1016/S13882457(00)00386-2

Koob, G. F., Caine, B., Markou, A., Pulverinti, L., \& Weiss, F. (1994). Role for the mesocortical dopamine system in the motivating effects of cocaine. NIDA Research Monograph, 145, $1-18$.

Littel, M., Euser, A. S., Munafò, M. R., \& Franken, I. H. A. (2012). Electrophysiological indices of biased cognitive processing of substance-related cues: A meta-analysis. Neuroscience \& Biobehavioral Reviews, 36, 1803-1816. doi: 10.1016/j.neubiorev.2012.05.001.

Littel, M., \& Franken, I. H. A. (2007). The effects of prolonged abstinence on the processing of smoking cues: An ERP study among smokers, ex-smokers and never-smokers. Journal of Psychopharmacology, 21, 873-882. doi: 10.1177/0269881107078494 
Littel, M., \& Franken, I. H. A. (2011). Implicit and explicit selective attention to smoking cues in smokers indexed by brain potentials. Journal of Psychopharmacology, 25, 503-503. doi: $10.1177 / 0269881110379284$.

Luck, S. J. (2005). An introduction to the event-related potential technique. Cambridge, MA: MIT Press.

Marshall, P. J., \& Meltzoff, A. N. (2011). Neural mirroring systems: Exploring the EEG mu rhythm in human infancy. Developmental Cognitive Neuroscience, 1, 110-123. doi: 10.1016/j.den.2010.09.001.

McFarland, D. J., Minder, L. A., Vaughan, T. M., \& Wolpaw, J. R. (2000). Mu and beta rhythm topographies during motor imagery and actual movements. Brain Topography, 12, 177186. doi: 10.1023/A:1023437823106.

Mogg, K., Bradley, B. P., Field, M., \& De Houwer, J. (2003). Eye movements to smokingrelated pictures in smokers: Relationship between attentional biases and implicit and explicit measures of stimulus valence. Addiction, 98, 825-836. doi: 10.1046/j.1360.200.00392.x

Muthukumaraswamy, S. D., Johnson, B. W., \& McNair, N. A. (2004). Mu rhythm modulation during observation of an object directed grasp. Cognitive Brain Research, 19, 195-201. doi: 10.1016/j.cogbrainres.2003.12.001

Oberman, L. M., Hubbard, E. M., McCleery, J. P., Altschuler, E. L., Ramachandran, V. S., \& Pineda, J. A. (2005). EEG evidence for mirror neuron dysfunction in autism spectrum disorders. Cognitive Brain Research, 24, 190-198. doi:

10.1016/j.cogbrainres.2005.01.014 
Oberman, L. M., Pineda, J. A., \& Ramachandran, V. S. (2007). The human mirror neuron system: The link between action observation and social skills. Social Cognitive and Affective Neuroscience, 2, 62-66. doi: 10.1093/scan/ns1022

Pineda, J. A. (2005). The functional significance of mu rhythms: Translating "seeing" and “hearing” into doing. Brain Research Reviews, 50, 57-68. doi:

10.1016/j.brainresrev.2005.04.005

Pineda, J. A., \& Hecht, E. (2008). Mirroring and mu rhythm involvement in social cognition: Are there dissociable subcomponents of theory of mind? Biological Psychology, 80, 306-314. doi: 10.1016/j.biopsycho.2008.11.003.

Pineda, J. O. A., \& Oberman, L. M. (2006). What goads cigarette smokers to smoke? Neural adaptation and the mirror neuron system. Brain Research, 1121, 128-135. doi:

10.1016/j.brainres.2006.08.128

Pineda, J. A., Silverman, D. S., Vankov, A., \& Hestenes, J. (2003). Learning to control brain rhythms: Making a brain-computer interface possible. IEEE Transactions on Neural Systems and Rehabilitation Engineering, 11, 181-184.

Polich, J. (2012). Neuropsychology of P300. In S. J. Luck \& E. S. Kappenman (Eds.), Handbook of event-related potential components (pp. 159-188), New York, NY: Oxford University Press.

Polich, J., \& Kok, A. (1995). Cognitive and biological determinants of P300: An integrative review. Biological Psychology, 41, 103-146. doi: 10.1016/0301-0511(95)05130-9

Polich, J., \& Ochoa, C. J. (2004). Alcoholism risk, tobacco smoking, and P300 event-related Q9 potential. Clinical Neurophysiology, 115, 1374-1383. doi: 10.1016/j.clinph.2004.01.026 
Pomerleau, O. F., Fagerström, K. O., Marks, J. L., Tate, J. C., \& Pomerleau, C. S. (2003).

Development and validation of a self-rating scale for positive- and negativereinforcement smoking: The Michigan Nicotine Reinforcement Questionnaire. Nicotine \& Tobacco Research, 5, 711-718. doi: 10.1080/1462220031000158627.

Tiffany, S. T. (1990). A cognitive model of drug urges and drug-use behavior: Role of automatic and nonautomatic processes. Psychological Review, 97, 147-168. doi: 10.1037/0033295X.97.2.147

Tiffany, S. T., \& Drobes, D. J. (1991). The development and initial validation of a questionnaire on smoking urges. British Journal of Addiction, 86, 1467-1476. doi: 10.1111/j.13600443.1991.tb01732.x

Townshend, J. M., \& Duka, T. (2001). Attentional bias associated with alcohol cues: Differences between heavy and occasional social drinkers. Psychopharmacology, 157, 67-74. doi: $10.1007 / \mathrm{s} 002130100764$

Urgesi, C., Moro, V., Candidi, M., \& Aglioti, S. M. (2006). Mapping implied body actions in the human motor system. The Journal of Neuroscience, 26, 7942-7949. doi: 10.1523/ JNEUROSCI.1289-06.2006.

Vadhan, N. P., Carpenter, K. M., Copersino, M. L., Hart, C. L., Foltin, R. W., \& Nunes, E. V. (2007). Attentional bias towards cocaine-related stimuli: Relationship to treatmentseeking for cocaine dependence. American Journal of Drug and Alcohol Abuse, 33, 727736. doi: $10.1080 / 00952990701523722$

Warren, C. A., \& McDonough, B. E. (1999). Event-related brain potentials as indicators of smoking cue-reactivity. Clinical Neurophysiology, 110, 1570-1584. doi: 10.1016/S13882457(99)00089-9 
Waters, A. J., \& Feyerabend, C. (2000). Determinants and effects of attentional bias in smokers. Psychology of Addictive Behaviors, 14, 111-120. doi: 10.1037/0893-164X.14.2.111

Waters, A. J., Shiffman, S., Sayette, M. A., Paty, J. A., Gwaltney, C. J., \& Balabanis, M. H. Q9 (2003). Attentional bias predicts outcome in smoking cessation. Health Psychology, 22, 378. doi: 10.1037/0278-6133.22.4.378

Yang, C. Y., Decety, J., Lee, S., Chen, C., \& Cheng, Y. (2009). Gender differences in the mu rhythm during empathy for pain: An electroencephalographic study. Brain Research, 1251, 176-184. doi: 10.1016/j.brainres.2008.11.062

Id\(RECEIVED October 15, 2012; ACCEPTED February 20, 2013) 
Itc|Table 1. Participant Characteristics

\begin{tabular}{lll}
\hline & tch $\backslash$ Smoker & Nonsmoker \\
& $(n=22)$ & $(n=20)$ \\
\hline tb\Age (in years) & $19.68 \pm 0.27$ & $18.70 \pm 0.20^{*}$ \\
Gender (\% female) & 45.5 & 30.0 \\
Ethnicity (\% Hispanic or Latino) & 4.5 & 0 \\
Race (\%) & & \\
African American & 4.5 & 25.5 \\
Asian & 9.0 & 0 \\
Caucasian & 86.4 & 75.0 \\
Carbon monoxide reading (ppm) & $3.36 \pm 0.59$ & $1.65 \pm 0.13 * *$ \\
Number of cigarettes smoked/day & $4.41 \pm 0.79$ & $0 *$ \\
& $3.00 \pm 0.58$ & $0 * *$ \\
\hline
\end{tabular}

ItfnWote. Values are presented as mean \pm standard error unless otherwise specified. ${ }^{*} p<0.05 .{ }^{* *} p<0.01$. 


\section{Figure captions}

Ifl\Figure 1. Examples of smoking-related and nonsmoking-related stimuli used in the current study. The pictures on the left represent the smoking condition, while the pictures on the right represent the nonsmoking condition. The top row shows active pictures while the bottom row shows inactive pictures.

\fl\Figure 2.

Ifl\Figure 3. Mu suppression to (A) smoking- and (B) nonsmoking-related cues as a function of image content, stimulus type, and participant status. *significantly less $(p<.05)$ mu suppression as indicated by simple main effects analyses. 